\title{
Rehabilitation: some thoughts on progress
}

\author{
I Bromley MBE MCSP
}

London, UK.

Over the past decade the attitude of the man in the street to medical practice has become more critical and demanding. He wants to be informed and involved in the choice of treatment. As a result the relationship between health workers and patients is becoming less autocratic and more of a partnership. This is reflected in the care of patients with spinal cord injury. For example, educational programmes provide information for patients and carers and encourage discussion of common problems in an increasing number of spinal units.

The psychological adjustment of those with spinal cord lesions has received less attention than their physical rehabilitation. A number of research studies have highlighted this deficiency and although this imbalance is being redressed much remains to be done.

A variety of technological advances and greater collaboration with physiologists, bioengineers and engineers have made major contributions to the enhancement of independence for paralysed people. Diaphragmatic pacing and new methods of artificial ventilation have enabled patients with extremely high lesions to live outside hospital.

The manufacture of wheelchairs adjustable to the size and needs of the individual have extended the function of the most able patients with low lesions. For example, a patient with a lesion at T12 is able to go up and down stairs unaided in the high performance wheelchair. As importantly, it has increased independence for those with high lesions and those who are older or less fit. These chairs also encourage and facilitate participation in sport.

Accompanying the changes in wheelchair design corresponding advances have been made in the manufacture of materials and cushions which can be used for correcting sitting posture. The high performance wheelchair with improved seating may reduce the incidence of scoliosis or at least limit the severity of the deformity.

A number of orthoses have been developed which enable walking to be achieved with less energy cost than with conventional calipers. ${ }^{1}$ Although these allow patients with higher lesions to walk, activities are limited when the trunk and lower limbs are both supported by the orthosis and adults use them primarily for exercise.

Research has shown that in the western world few patients with complete lesions continue to walk outside hospital and less emphasis is now placed on gait training during the initial period of rehabilitation. However in countries where the terrain is unsuitable for wheelchairs, walking with calipers and crutches is vital if maximal independence is to be achieved. As more patients in these countries survive their initial injury, progress is being made in the acceptance of disabled people in the community. The necessity to learn to walk may therefore increase.

Where functional electrical stimulation (FES) has been developed, an additional option is available for those patients keen to walk, using either FES on its own or in combination with an orthosis. Although progress has been made in this field, as yet the number of patients who can really benefit is extremely small. ${ }^{2}$ Perhaps FES should be developed particularly for patients in those countries where it is difficult to use a wheelchair and walking is essential?

Little is known about the effects of spending years in a wheelchair, putting unnatural stress upon the joints of the trunk and upper limbs. Research currently being undertaken will particularly interest therapists as results may indicate changes in relevant rehabilitation goals. 
With the high level of skilled care now so often provided at the site of the accident, a greater number of spinal injuries result in incomplete lesions. The need to make the best use of muscles hampered by spasticity has been challenging therapists for many years. There are different approaches to treatment and various claims for success are made. However, little research has been undertaken and this will need to be remedied if the most efficient and effective use is to be made of scarce resources.

The aim of rehabilitation is to prepare the individual to return to life at home, yet the development of appropriate facilities has been slow. Inequalities in social provision continue to exist between nations, and even between different parts of the same country. Patients with lesions at $\mathrm{C} 2$ can be provided with all the assistance and equipment they require to live at home in some countries whilst in others, few even with low lesions can return home as housing and relevant equipment are unavailable. To add to the difficulties, therapists are unequally spread around the world. In those countries where there are few facilities to enable disabled people to live at home, the ratio of therapists to the population is also low and most work in hospitals. In some of these countries, teams of rehabilitation workers are being trained to work locally in their own communities. These initiatives are often frowned upon by professional personnel with traditional attitudes and idealistic expectations for care which can never be realised.

Where the technology is available robots are being developed to perform certain tasks and assist severely handicapped people to live at home. Animals such as dogs and monkeys are being trained to do similar tasks. These schemes are in their infancy and their feasibility and worth will need to be very carefully evaluated.

Research in rehabilitation, where so many disciplines make a contribution, is not easy. The current emphasis on defining quality and developing methods for multidisciplinary audit, where these do not already exist, may help to pinpoint priorities for research. Perhaps physiotherapists and other health care staff, working with patients with spinal cord lesions, could adopt the procedure used by physiotherapists to identify milestones in the recovery of patients with stroke. ${ }^{3}$ In this study monitoring recovery over an 8-week period showed a distinct time related pattern. A useful baseline in rehabilitation could be provided by patterns of this sort. As the researchers state, it would allow the 'comparisation of individual scores with an average for that phase of the illness, the setting of precise goals and the examination of factors that influence recovery' in addition to 'examining the relations between disability, actual limitation of movement and handicap in daily life.'

In such a relatively small field and where comparatively little research has been undertaken maybe the next decade will see greater mutual trust between rehabilitation workers and implementation of each other's research findings. With the development of specialisation and research in the rehabilitation of those with spinal cord injuries, there will be a need for communication and an awareness of the breadth of research undertaken. For example, those involved in training sportsmen with tetraplegia need to know about recent research in Sweden showing that the diaphragm acts as a postural muscle. Training tetraplegics for wheelchair marathons may overwork the diaphragm and be potentially dangerous. ${ }^{4}$

The value of international exchange between certain members of the staff of spinal units has already been promoted and supported. However, this has been a rarity for therapists. Considering the amount of time spent in the complex process of rehabilitation, more opportunities for them to learn from each other would benefit patients worldwide.

\section{References}

1 Nene AV, Patrick JH (1989) Energy cost of paraplegic locomotion with the Orlau walker Paraplegia 27(5): 5-18.

2 Jaeger RJ, Yarkony GM, Roth EJ, Lovell BS (1990) Estimating the user population of a simple electrical 
stimulation system for standing. Paraplegia 28(5): 505-511.

3 Partridge CJ, Johnston M, Edwards S (1987) Recovery from physical disability after stroke: normal patterns as a basis for evaluation. Lancet February I4: 373-375.

4 Sinderby CP, Invarsson L, Sullivan I, Wickstrom, Lindstrom L (in press) The role of the diaphragm in trunk extension in tetraplegia. Paraplegia. 